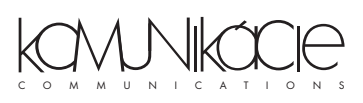

\title{
DYNAMIC AIR TRAFFIC CONTROL WAKE VORTEX SAFETY AND CAPACITY SYSTEM
}

International regulations require aircraft to be separated in approach phase of flight by up to six nautical miles (11.12 km) due to the potential hazard caused by the swirling air left in their wakes. This 'wake vortex' is now the subject of intense worldwide research to understand the nature of the phenomenon and find ways of making air travel safer while reducing congestion around airports. "With the world airline fleet expected to double in size over the next 15 years and the giant A380 entering service in 2005, solutions to the wake-vortex problem cannot come too soon for the aerospace industry and therefore there is a strong need for developing a dynamic air traffic control wake vortex safety and capacity system." [1] This paper describes the concept of integrated transportation system regarding the current state-of-the-art technologies.

\section{Introduction}

Just as a ship leaves a wave behind it in the sea, an aircraft leaves a wake in the air. An aircraft's wake is in the form of two counter-rotating swirling rolls of air - the wake vortices - that trail from the wings of the aircraft. The wake vortex pair may last for several minutes and may stretch for many kilometres behind the aircraft. The strength of the vortices basically depends on the aircraft weight, divided by the product of air density, flying speed and wingspan. This property generally increases with aircraft weight. The lifetime of a vortex depends upon local meteorological conditions. Vortices last longer in calm air and atmospheric turbulence hastens their decay.

Why do wake vortices matter? It is a question of safety. The rapidly swirling air in a vortex can catch the wings of a following aircraft with potentially disastrous results. Tests with experienced test pilots have shown that even heavy size commercial airliners can be thrown out of control if they follow too close behind a large aircraft such as a Boeing 747. Wake vortices are normally invisible and pilots have no warning that they are flying into one. For this reason, the International Civil Aviation Organization (ICAO) lays down strict rules about the permitted spacing between aircraft, based on their size. In instrument flying conditions aircraft may follow no closer than three nautical miles $(5.56 \mathrm{~km})$, and a small aircraft must follow at least six nautical miles $(11.12 \mathrm{~km})$ behind a heavy jet such as a Boeing 747 .

Many airline pilots have had encounters with vortices, usually on the final approach to airports. They are experienced as a buffeting of the aircraft. While of little concern to passengers and crew who are wearing seat belts at this stage, pilots regularly report minor injuries to crewmembers standing up or moving around the cabin. However, thanks to ICAO regulations on separations (Fig. 1), there have been no serious accidents reported with passenger air-

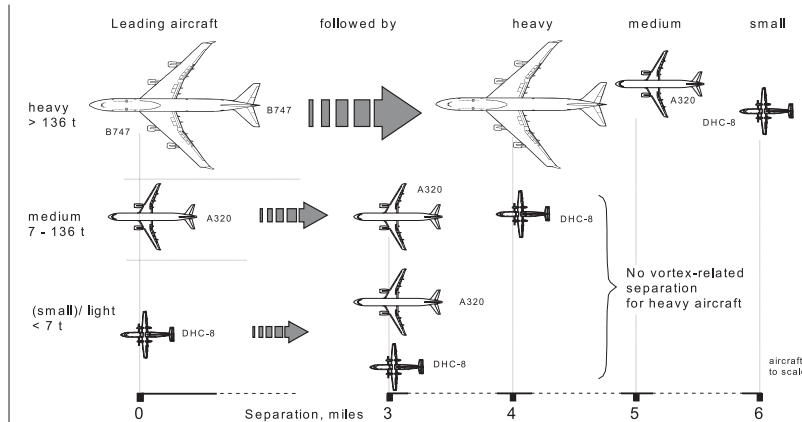

Fig. 1. ICAO separation scheme for single runway approaches

liners until November 12 2001, when an Airbus 300 crashed in New York and one of the mentioned reasons was the wake encounter.

"ICAO separations are conservative: they do not completely avoid the effects of wake vortices, but they are sufficient to be safe in most meteorological conditions." [2] Particularly noteworthy is that appropriate regulation for closely spaced parallel runways (separated by less than $2500 \mathrm{ft}$ ) is lacking, resulting in inefficient use of some of the runway configurations. The present regulation prescribes that such runways must be used as single runways when the spacing is less than $2500 \mathrm{ft}$ (or $760 \mathrm{~m}$ ) and in case of instrument meteorological conditions (IMC). Since building an additional closely spaced parallel runway at existing European airports is often the only possible feasible extension possibility, this matter is of crucial importance to increase airport capacity.

Since new high capacity aircraft (such as the Airbus A380) will be heavier and larger, and air traffic has grown continuously with an average rate of $4 \%$ per year, today's aircraft separation rules are considered increasingly inefficient, and may result in unnecessary delays. An integrated Air Traffic Control (ATC) wake vortex safety and capacity system (including a controller Human Machine

\footnotetext{
* Peter Choroba

This work was supported by Eurocontrol Experimental Centre. The author is with the Eurocontrol Experimental Centre, Centre des Bois des Bordes, B.P. 15, F-91222 Bretigny sur Orge Cedex, France (Tel: +33-1-6988-7858; E-mail: peter.choroba@eurocontrol.int).
} 
Interface (HMI)) used in combination with new modified wake vortex safety regulation is expected to provide the means to significantly enhance airport capacity, basically on runway throughput.

\section{The components of the system}

The impact of weather on wake vortex safety is a crucial aspect, and the uncertainty in predicting the behavior of wake vortices in different weather conditions implies that continuous monitoring of both wake vortices and weather will be necessary. This will enable continuous verification - and possibly update - of safe predictions of required aircraft spacing (separation minima). The integrated wake vortex safety and capacity system should therefore integrate the following subsystems:

- Wake detection sensors

- Weather forecast and now cast systems

- Wake vortex prediction tool

- Air Traffic Controller Human Machine Interface (HMI)

\section{A. Wake detection sensors}

The basic requirements for a wake vortex sensor are to detect, locate and quantify the strength of aircraft wake vortices. There are several tools (systems), which enable detection of wake vortices with different limitations in range, accuracy, and weather sensibility. The simplest one is WINDLINE, based on mechanical approach of vortex detection by set of anemometers. This system is highly accurate, but cannot be used for higher altitudes, since anemometers are placed on the ground. On the other hand, advantage of this sensor is usability in all weather conditions.

Vortices can be detected by ground-based or in the future by onboard-pulsed lidars. LIDAR (LIght Detection And Ranging) is similar to the more familiar radar and can be thought of as laser radar. In radar radio waves are transmitted into the atmosphere, which scatters some of the power back to radar's receiver. LIDAR

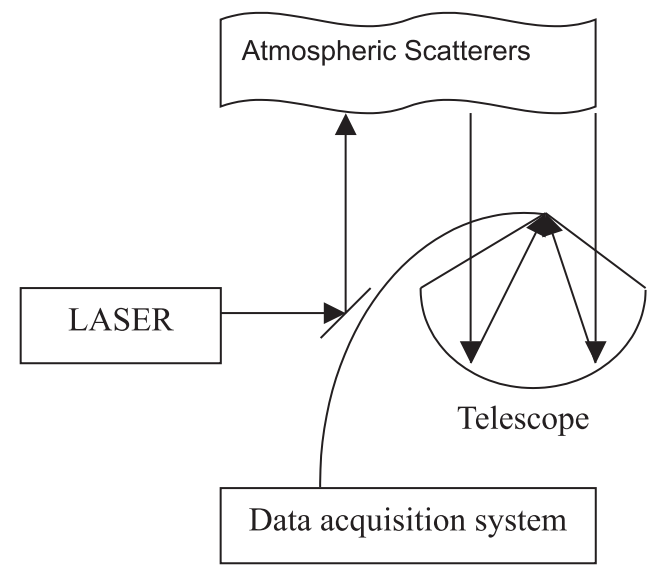

Fig. 2. Schematic view of LIDAR's architecture transmits and receives electromagnetic radiation but at a higher frequency (ultraviolet, visible and violent region). Different types of physical processes in the atmosphere are related to different types of light ring. Choosing different types of scattering processes allows atmospheric composition, temperature and wind to be measured. LIDAR in general consists of 3 main parts: transmitter, receiver and detector system (Fig.2).

LIDARs use extremely sensitive detectors so called photo multiplier tubes. An individual quantum of light is converted first into electric currents and then into digital photo counts, which can be stored and processed on a computer. The received photo counts are recorded for fixed time intervals during the return pulse. The times are converted to heights called range bins since the speed of light is well known. The range-gated photo counts are then stored and analysed by a computer.

"CW (continuous wave) lidar has the best range resolution but is limited to about 300 meters in range, so it used close to the runway threshold. The pulsed lidar can measure wakes out to several kilometers, but has poorer resolution ( $~ 30$ meters)." [3] However, LIDAR capability in rain and fog has not been demonstrated.

The SODAR (Sound detection and ranging) may also be capable to detect wake vortex. The newest SODAR systems use steered beam, phased array technology. Three independent beams are generated: one directed vertically and two tilted in perpendicular directions. Their compact design is ideal for measuring and providing high-resolution wind and wind turbulence profiles from a wide variety of platforms such as ships, buildings, trailers and trucks. The SODAR acoustic signal processor is engineered for reliable, unattended field operation. Data analysis software is also available to process SODAR data, including automatic generation of data reports and summary plots. SODAR's height range is depending on the model in interval 15 - 750 meters, with resolution 5- $20 \mathrm{~m}$

A building block necessary to build sufficient confidence in the model predictions for wake vortices evolution in relation to weather will be to use data from a C-Band Doppler weather radar for weather monitoring but also wake vortex detection. Several US references in scientific literature put forward the idea that a Doppler radar can detect wake vortices, and it must be stated that currently some research centers are starting to use C-band Doppler radar data as experimental trials relevant data. The interest of radar for weather analysis is that of a system complementary to the lidar system:

- It can provide additional meteorological parameters besides performing turbulences detection (especially for mapping of precipitations areas where lidar is not efficient)

- The potential detection range on wake vortex is at least the same as for lidar in clear air, but is larger in humid air and in rain.

Principles and algorithms (for e.g. atmospheric turbulence detection and weather forecasting) previously developed within three European studies of the 4th Research Technology and Devel- 
opment Framework Program. These algorithms improve significantly the Doppler radar analysis performances and are well adapted for wake vortex monitoring. Some principles have already been validated on low altitude wind-shears detection.

Nevertheless, the limitations as well as uncertainties in measurements of wake turbulence detections are yet to be evaluated as most technologies are still under development.

\section{B. Weather forecast and now cast systems}

Since the weather impact on wake vortex behavior is significant, the weather forecast and now cast must be provided as one the required data sets for further calculations, especially for vortex prediction computing. Weather subsystem is crucial and must provide detailed wind, vertical wind shear, atmospheric turbulence and temperature gradient information to the prediction subsystem for the current time and up to an hour in the future. Besides the common used airport weather radars (mostly C-Band Doppler radars) it is necessary to use wind profilers to get accurate wind and temperature profiles.

"Wind profilers are pulse-Doppler radars that look into the atmosphere and make high-resolution measurements of wind, turbulence, and echo strength in a vertical column above the radar site." [4] Typically, wind measurements are made by pointing the radar's antenna beam in three (or sometimes five) different directions. The Doppler shifts of the echoes from each direction are compared to determine the wind speed and direction. Profilers can operate continuously, providing wind measurements approximately every 5 minutes. The scattering targets from which echoes are received depend on the wavelength used. In the case of UHFband profilers, the targets include rain, snow, and turbulent fluctuations (causing changes in refractive index) of clear air. Some profilers can also measure temperature using a Radio Acoustic Sounding System (RASS) in which sound waves are also emitted and tracked by the radar head. The measured speed of the sound is related to air temperature.

The effective altitude range of a profiler depends on the wavelength used and other factors such as the antenna size, transmitter power, and the availability of scattering targets. "For a typical profiler, the range in fair weather is from about one half to five kilometers during the summer, although on cold, clear, winter days sometimes no measurements can be made at all." [4] In rain or snow, measurements can be made from as low as 100 meters and often up to ten- $\mathrm{km}$ altitude or higher. These profilers are often called boundary layer profilers because of the region in which they are most effective. Other profilers, operating in the VHF band, are designed to make measurements higher in the atmosphere; some, for example, probe the mesosphere, stratosphere, and troposphere.

\section{Wake vortex prediction tools}

The core of the integrated system is the prediction subsystem. The predictor utilizes weather data and an aircraft characteristic database to predict the wake and threshold of wake vortex strength for an acceptable encounter, airport layout data and wake sensor feedback.

Two most capable prediction tools in Europe nowadays are: P2P developed by DLR (Deutsches Zentrum fur Luft- und Raumfahrt) and Vortex Forecast System (VFS) developed by Transport Canada but with European cooperation.

"A new parametric wake vortex transport and decay model is proposed that predicts probabilistic wake vortex behavior as a function of aircraft and environmental parameters in real-time." [5] The Probabilistic Two-Phase wake vortex decay model (P2P) accounts for the effects of wind, turbulence, stable stratification, and ground proximity. The model equations are derived from the analytical solution of the spatial-temporal circulation evolution of the decaying potential vortex and are adapted to wake vortex behavior as observed in large eddy simulations. Vortex decay progresses in two phases, a diffusion phase followed by rapid decay. Vortex descent is a non-linear function of vortex strength. Probabilistic components account for deviations from deterministic vortex behavior inherently caused by the stochastic nature of turbulence, vortex instabilities and deformations, as well as uncertainties and fluctuations that arise from environmental and aircraft parameters. The output of P2P consists of confidence intervals for vortex position and strength.

To reliably assign a defined degree of probability to the predictions, the model design allows you for the continuous adjustment of decay parameters and uncertainty allowances, based on a growing amount of data. "The application of a deterministic version of P2P to the Memphis wake vortex database yields favorable agreement with measurements." [6]
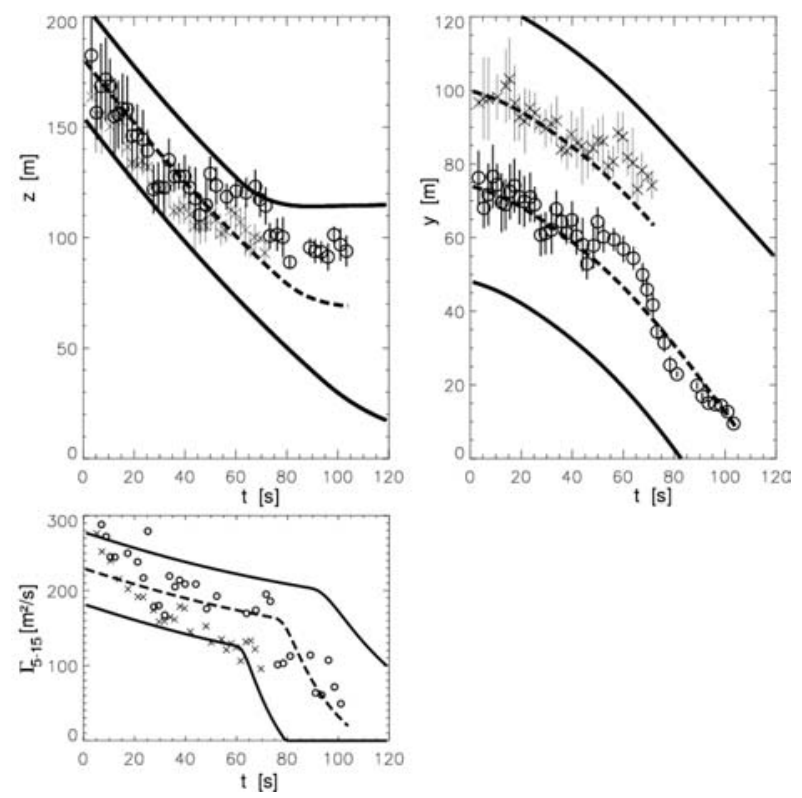

Fig. 3. Comparison of prediction and detection (DLR project Wirbelschleppe) 
Comparison between wake vortex prediction made by $\mathrm{P} 2 \mathrm{P}$ and measurements conducted by LIDAR is shown in Fig. 3. The bold lines present the predicted confidence interval in vertical $(z)$ and lateral $(y)$ plane, as well as circulation $(\Gamma)$ data. Measurements were made by two lidar systems.

"Vortex Forecast System (VFS) is a prediction tool based on a combination of theory (method of discrete vortices) and experimental data. Its accuracy and speed in predicting potentially dangerous wake vortices may allow the safe distances between aircraft to be reduced, thus increasing capacity of congested airport." [7]

VFS uses real-time information about the environment and the aircraft, predicted meteorological conditions, and accurate real-time modeling of vortex transport and decay to predict conditions under which the separation may be safely reduced below the current wake vortex standards. The principal components of VFS are the Near Wake, Far Wake, and Danger Area Models. The Near Wake Database (NWDB) is calculated offline for each aircraft type based on the information available on the aircraft geometry. The far wake evolution is calculated in real time. The far wake calculation may start from the NWDB, or alternately from a universal near wake profile. The far wake calculations are based on the 2-D crossplane method of discrete vortices equations describing the vortex motion in incompressible flows.

\section{Air Traffic Controller Human Machine Interface (ATC HMI)}

The role of the ATC HMI is to provide the traffic situation picture and automated support for various ATC tactical roles e.g. approach, tower. It is foreseen to integrate wake vortex related information together with flight information (position, altitude, ground speed, aircraft type) in order to improve the safety and capacity. Current ATC systems do not take into account wake turbulence information, hence controllers rely on the ICAO separation standards or very often just on their own experience. The AVOSS (Advance vortex spacing system) study shows [8], that in specific weather conditions even the ICAO separations are not enough and there is a potential of wake vortex encounter. On the other hand, separations are quite often too conservative, thus limiting capacity. Wake vortex information visualization can be integrated in any of the current air traffic control human machine interfaces and can be used for optimization of separations.

HMI has to be developed and optimized for tower and Terminal Area / en-route controllers, under the commitment to the principles of human centered automation. That means, with priority to providing optimal decision support to the controllers, who will keep the ultimate responsibility for their decisions, HMIs will have to reflect a synthesis between

- Specific controller needs for information and decision support

- Usability and acceptability of the HMIs

- Airport operational requirements and constraints

- Traffic demands (e.g. amount of inbound/outbound traffic)
- Technical functionality provided by the integrated system, particularly for wake vortex prediction and monitoring, and aircraft spacing prediction.

A further issue is merging those functionalities with other functions at the controller working positions (e.g. approach planning, departure planning). This issue has to be addressed through an analysis of the interoperability with existing ATC systems and the usability and acceptability of the system.

\section{Sensor and data fusion}

To develop an integrated wake vortex safety and capacity system it is necessary to combine all the components mentioned above and to deal with the data fusion. Fig. 4 illustrates the data flow.

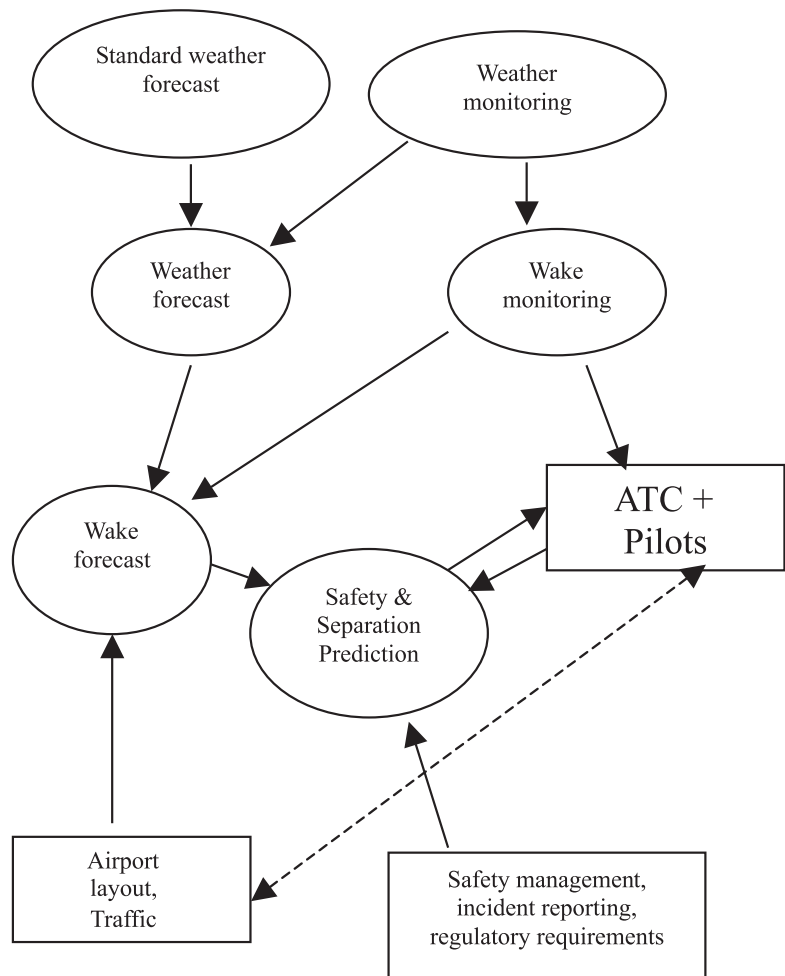

Fig. 4. Diagram of data flow in the integrated system

Standard weather forecast data are used as input for local weather forecast at the particular airport using also data from weather monitoring at the same airport. Wake monitoring (detection) requires also input from local weather monitoring subsystem and also provides wake information to pilots and air traffic controllers, especially in the case of detected very strong wake turbulence to avoid an encounter.

Wake vortex prediction is computed with an input of airport layout and traffic data including weather forecast and wake moni- 
toring data. After the wake prediction, safety and separation prediction can be computed regarding to safety management, incident reporting and regulatory requirements. Output of this step provides "new" spacing criteria to controllers and pilots.

Integration of the heterogeneous subsystems requires state-ofthe-art facilities, including a variety of methods for integration (e.g. static/dynamic interaction, tool chaining, workflow chains). Design of the integrated platform comprises the design of system architecture, interfaces, common database, scenario manager, and humanmachine interfaces (including controller HMI).

\section{Conclusion}

The local installation of the integrated system at the airports will require new safety regulation, since the present wake vortex safety recommendations and best practices do not take new modified ATC systems into account. Specific attention must be given to the issue of development and harmonization of new wake vortex safety regulation. To enhance acceptability of the integrated system (and other new technologies, including high capacity aircraft such as the Airbus A380 and on-board wake detection and warning instrumentation), possible end-users and regulatory authorities should be involved in the development of such system to achieve the goal as soon as possible. The concept of the integrated system will be validated firstly through the fast-time simulation followed by real-time simulation, including assessment of safety and capacity benefit.

\section{References}

[1] EUROPEAN COMMISSION RESEARCH PROJECTS OVERVIEW: Wake vortex, 2002

[2] GERZ, T., HOLZAPFEL, F, DARRACQ, D.: Aircraft wake vortices (A position paper), 2001

[3] HINTON, D. A.: An aircraft vortex spacing system (AVOSS), NASA Langley, 2001

[4] CARTER, L. A.: Developments in UHF lower troposphere wind profiling at NOAA, 1995

[5] HOLZAPFEL, F.: A probabilistic two-phase wake vortex decay and transport model, DLR 2001

[6] HOLZAPFEL, F., ROBINS, E. R.: Probabilistic wake vortex decay model predictions compared with observations, DLR 2002

[7] JACKSON, W.: Wake vortex prediction - an overview, Transport Canada 2001

[8] Wake vortex measurements data - DFW Deployment of September 15 to October 3, 1997

Peter Choroba is a Ph.D. student in the Innovative R\&D of Eurocontrol Experimental Centre in Bretigny sur Orge/Paris. He holds a Master in Air Transportation engineering from the University of Zilina in the Slovak Republic. 